\title{
DYNAMICS OF CONTROLLED HYBRID SYSTEMS OF AERIAL CABLE-WAYS*
}

\author{
Olena V. Mul ${ }^{1) \dagger} \quad$ Delfim F. M. Torres ${ }^{1)}$ \\ Volodymyr P. Kravchenko 2 ) \\ 1) Department of Mathematics, University of Aveiro \\ Campus Universitário de Santiago \\ 3810-193 Aveiro, Portugal \\ Email: \{olena, delfim\}@mat.ua.pt \\ 2) Physico-Technological Institute of Metals and Alloys \\ of the National Academy of Sciences of Ukraine \\ Academician Vernadsky Avenue, 34/1 \\ 03680 Kiev-142, Ukraine \\ Email: v_kram@i.com.ua
}

\begin{abstract}
Dynamics of the hybrid systems of aerial cable-ways is investigated. The eigenvalue problems are considered for such hybrid systems with different assumptions. An overview of different methods for eigenvalue problems is given. In the research, the method of the normal fundamental systems is applied, which turns out to be very effective for the considered problems. Changes of dynamical characteristics of the systems depending on the controlled parameter are studied.
\end{abstract}

Mathematics Subject Classification 2000: 37N35, 35B37, 93C20.

Keywords: hybrid systems, eigenvalue problems, numerical methods, normal fundamental functions.

\section{Introduction}

Non-stationary boundary problems with continuous-discrete parameters in the theory of vibrations belong to the wide class of continuous-discrete boundary

\footnotetext{
*Accepted (15-May-2006) to the Proceedings of the International Conference of Hybrid Systems and Applications, The University of Louisiana, Lafayette, LA, USA, May 22-26 2006, to be published in the journal Nonlinear Analysis: Hybrid Systems and Applications.

†Supported by FCT (the Portuguese Foundation for Science and Technology), fellowship SFRH/BPD/14946/2004.
} 
problems, whose parameters change with time. These problems are of great importance both theoretically and in applications [4. Typically, their study combines solving non-stationary problems with continuous parameters [6, 7, 10] and non-stationary problems with continuous-discrete parameters 4, 8.

The dynamics for many elastic systems is described by partial differential equations with non-stationary continuous-discrete coefficients. Moreover, the solutions of those equations have to satisfy not only some boundary conditions but also some conjugation conditions, which considerably complicate the analytical or numerical study. The use of normal fundamental functions 3 allows to find recurrence formulas, by means of which the conjugation conditions are taken into account. This simplifies considerably the resolution of the non-stationary continuous-discrete boundary problems.

In this paper dynamics of some controlled systems of aerial cable-ways with continuous and discrete parameters is studied. For research, mathematical models in the form of the eigenvalue problems are used. In $\$ 2$ for similar eigenvalue problems it is given an overview of different known methods, such as variational methods, the Raleigh's energy method and methods of integral equations. In \$3 an application of the method of the normal fundamental systems, which turns out to be the most effective one for the hybrid eigenvalue problems, is described. In 4 we consider several eigenvalue problems that model the dynamics of aerial cable-ways at different assumptions about the system which is: only the cable without discrete loads, the cable with fixed loads, the cable with loads moving along it at a steady speed, or the cable with fixed discrete loads moving at a steady speed. The method of the normal fundamental systems is applied to these problems, and the dependence of the dynamical characteristics of the systems on the controlled parameter, which is the speed of the system motion, is studied.

\section{An Overview of Methods for Eigenvalue Prob- lems}

The mathematical problems on eigenvalues are widespread both in theoretical and applied mathematics. They serve as models for many concrete problems in physics, engineering and biology. Most of these applied problems may be reduced to the study of boundary problems for ordinary or partial differential equations, where almost always the main question is reduced to the determination of the eigenvalues and eigenfunctions [1].

There exist many different non-stationary boundary problems with continuous and discrete parameters which are mathematical models for a wide class of controlled dynamical systems [4. Dynamical characteristics of such hybrid systems may be controlled by changing the system parameters within certain limits. Vibrations in such systems are described both by partial differential equations with variable coefficients and by homogeneous Fredholm integral equation, with a Stieltjes type of integral. 
In order to determine the eigenvalues of the vibration boundary problems, different variational methods, as well as methods of differential and integral equations, may be used [2, 5].

An eigenvalue problem may be reduced to a variational problem, viz, to the search of the minimum of a certain functional [3]. One of the most well-known variational method is the Ritz method, which can be applied to the problem

$$
\min _{u}(A u, u)
$$

subject to the additional condition

$$
(u, u)=1,
$$

where $A$ is a positive operator and $u$ is a continuous function. In order to solve problem (11)-(2) by the Ritz method, one begins by choosing some sequence of basic functions $\varphi_{n}, n=1,2, \ldots$. They have to satisfy the following three conditions: all functions $\varphi_{n}$ belong to the operator definition domain, they are linearly independent for any $n$, and their system $\left\{\varphi_{n}\right\}$ is complete. Then, one searches the approximate solution in the form

$$
u_{n}=\sum_{k=1}^{n} a_{k} \varphi_{k},
$$

where the $a_{k}$ 's are unknown coefficients, with $k=\overline{1, n}$. The coefficients $a_{k}$ 's should be selected in a way to satisfy condition (2) for the solution $u_{n}$ and to make the value $\left(A u_{n}, u_{n}\right)$ minimal. The Ritz method can be applied only to continuous problems, and is not applicable to the problems with continuous and discrete distribution of masses we are interested in.

There exist many other variational methods, for example, the BubnovGalerkin method, which is a generalization of the Ritz approach for operators that are not obligatory positive. However, an application of the BubnovGalerkin method for continuous-discrete problems results in significant difficulties on the choice of the coordinate functions for the complex domains. Other variational methods are also inappropriate to the study of continuous-discrete problems, because of the complexity of their application. Another disadvantage of all the variational methods is that the obtained approximations for the eigenvalues always exceed their real values.

The Raleigh's energy method is also usually applied to solve eigenvalue problems. The idea of this approach is as follows. If the mode of vibrations is known, we can always find the frequency of free vibrations from the constancy of the sum of the kinetic and potential energy. Then, with the known frequency, it is easy to find the eigenvalues. However, the problem is to choose the mode of vibrations. Raleigh proposed to take the shape of the system static deformation from the applied load as the first mode of vibrations. Since the selection of the next vibration mode is complicated, the Raleigh's method is convenient only for the determination of the first frequency and the first eigenvalue. This is not enough for our purposes. 
Eigenvalue problems may be also reduced to the determination of eigenvalues of integral equations of the form

$$
y(x)=\lambda \int_{a}^{b} r(x) G(x, s) y(s) d s,
$$

where $G(x, s)$ is the Green function; $r(x)$ is a continuous positive function; $y(x)$ is an unknown function; $\lambda$ is an unknown eigenvalue; $a$ and $b$ are known constants.

Equation (4) can be transformed to an integral equation with a symmetric kernel. The advantage of the eigenvalue problem method, which deals with the determination of eigenvalues and eigenfunctions in the case of a symmetric kernel, is that the same integral equations describe different vibration processes, such as flexural, torsional, longitudinal or flexural-and-torsional ones. Besides, in spite of the different physical sense for the Green's function, the integral equations are the same for the systems with continuous and with continuousdiscrete parameters. On the base of the integral methods it is possible to create an universal technique for solving eigenvalue problems for discrete and discretecontinuous systems. The big problem with the integral methods, which explains why they are not usually used in applications, is the great complexity in the construction of the Green's function.

Thus, most of the known methods for eigenvalue problems cannot be applied in an effective way to the case of continuous-discrete systems. In the next section we consider an efficient numerical method for such hybrid eigenvalue problems.

\section{The Normal Fundamental Function Method}

Here we briefly illustrate the normal fundamental function method by using the following system of partial differential equations with variable coefficients [9]:

$$
\begin{gathered}
\frac{\partial z_{k}}{\partial y}=\sum_{j=1}^{N} A_{k j}(y) z_{j}+\sum_{j=1}^{N} B_{k j}(y) \frac{\partial^{2} z_{j}}{\partial t^{2}}+\sum_{j=1}^{N} C_{k j}(y) \frac{\partial z_{j}}{\partial t} \\
y_{0} \leq y \leq y_{n}, \quad k=\overline{1, N}
\end{gathered}
$$

satisfying the linear homogeneous boundary conditions

$$
\begin{gathered}
z_{r}(0)=0, \quad r=\overline{1, m} \\
z_{s}(l)=0, \quad s=\overline{m+1, N}, \quad N=2 m,
\end{gathered}
$$


and the conjugation conditions

$$
\begin{aligned}
\sum_{k=1}^{N} a_{k, p}^{(i+1)}\left(y_{i}\right) z_{k}^{(i+1)}\left(y_{i}\right) & +\sum_{k=1}^{N} b_{k, p}^{(i+1)}\left(y_{i}\right) \frac{\partial^{2} z_{k}^{(i+1)}\left(y_{i}\right)}{\partial t^{2}} \\
+ & \sum_{k=1}^{N} d_{k, p}^{(i+1)}\left(y_{i}\right) \frac{\partial z_{k}^{(i+1)}\left(y_{i}\right)}{\partial t}=\sum_{k=1}^{N} a_{k, p}^{(i)}\left(y_{i}\right) z_{k}^{(i)}\left(y_{i}\right) \\
& +\sum_{k=1}^{N} b_{k, p}^{(i)}\left(y_{i}\right) \frac{\partial^{2} z_{k}^{(i)}\left(y_{i}\right)}{\partial t^{2}}+\sum_{k=1}^{N} d_{k, p}^{(i)}\left(y_{i}\right) \frac{\partial z_{k}^{(i)}\left(y_{i}\right)}{\partial t},
\end{aligned}
$$

where $z_{j}(y, t)$ is the deviation function of the vibration system from an equilibrium position; $A_{k j}(y), B_{k j}(y)$ and $C_{k j}(y)$ are real-valued piecewise continuous functions bounded on the interval $\left[y_{0}, y_{n}\right]$ for $k, j=\overline{1, N} ; y$ is the coordinate of the system point; $t$ is the time variable; $y=y_{i}$ are the discontinuity points of functions $A_{k j}(y), B_{k j}(y)$ and $C_{k j}(y)$ for $i=\overline{1, n-1} ; p=\overline{1, N}$; the superscript $i$ means the value of the corresponding function on the $i$-th interval.

The continuous-discrete boundary problem (5)-(7) describes a large number of different dynamical systems [3, 4].

For integration of system (5)-(7) we apply the Fourier method of separation of variables, searching the solution in the form

$$
z_{k}(y, t)=\varphi_{k}(y) e^{\lambda t} .
$$

We are interested in finding the values for the parameter $\lambda$, for which there exist non-trivial solutions $\varphi_{k}(y)$ of the system (5)-(17). Such values $\lambda$ are eigenvalues of the boundary problem, and the corresponding solutions $\varphi_{k}(y)$ are eigenfunctions. The eigenvalues for the vibration system, described by equations (5)-(7), are complex quantities $\lambda=q+i p$, where $i=\sqrt{-1}$. The vibrations have increasing or decreasing amplitude, depending on the sign of the real part $q$ of the eigenvalue.

In many practical vibration theory problems it is important to know eigenvalues at which vibrations with a constant amplitude occur. That is only possible at an imaginary eigenvalue $\lambda=i p$, where $p$ is the eigenfrequency of the vibrations of the considered system.

Thus, we assume that the system makes harmonic vibrations with constant amplitude and the form of solution is

$$
z_{k}(y, t)=\left[\varphi_{k}^{(1)}(y)+\varphi_{k}^{(2)}(y)\right] e^{i p t},
$$

where $\varphi_{k}^{(1)}(y)$ and $\varphi_{k}^{(2)}(y)$ are real-valued functions. Then the problem (5)-(7) is reduced to the normal system of linear ordinary differential equations

$$
\frac{d \varphi_{k}}{d y}=\sum_{j=1}^{N} a_{k j}(y) \varphi_{j}, \quad k=\overline{1, N}
$$


satisfying $N$ linear homogeneous boundary conditions and also linear conjugation conditions at the discontinuity points, where, for simplicity, $\varphi_{k}$ includes both $\varphi_{k}^{(1)}$ at the values $k=\overline{1, m}$ and $\varphi_{k}^{(2)}$ at the values $k=\overline{m+1, N} ; a_{k j}(y)$ are bounded piecewise-continuous functions on the interval $\left[y_{0}, y_{n}\right] ; k, j=\overline{1, N}$.

Now, for each interval $y_{i-1} \leq y \leq y_{i}, i=\overline{1, n}$, we can solve a Cauchy problem for the system (9) with the initial conditions at points $y=y_{i-1}$ as follows:

$$
\Phi^{(i)}\left(y_{i-1}\right)=E, \quad i=\overline{1, n},
$$

where $E$ is the unit matrix. According to Picard's Theorem, each such problem has a unique solution. We can apply some known numerical method, for example the Runge-Kutta method, to find $N$ linearly independent solutions of the system (9). Such fundamental system of solutions

$$
\Phi^{(i)}(y)=\left|\begin{array}{ccc}
\varphi_{1,1}^{(i)}(y) & \ldots & \varphi_{1, N}^{(i)}(y) \\
\ldots & \ldots & \ldots \\
\varphi_{N, 1}^{(i)}(y) & \ldots & \varphi_{N, N}^{(i)}(y)
\end{array}\right|, \quad y_{i-1} \leq y \leq y_{i}, \quad i=\overline{1, n}
$$

with initial conditions (10) is called a normal fundamental system [3]. Here each function $\varphi_{k, j}^{(i)}(y)$ is defined and continuous at $\left[y_{i-1}, y_{i}\right] ; k$ is a solution number; $j$ is a function number. The general solution of the system (9) at the $i$-th interval may be written with the help of the normal fundamental system of solutions as

$$
\varphi_{k}^{(i)}(y)=\sum_{j=1}^{N} C_{j}^{(i)} \varphi_{j, k}^{(i)}(y), \quad y_{i-1} \leq y \leq y_{i}, \quad k=\overline{1, N}, \quad i=\overline{1, n}
$$

where $C_{j}^{(i)}$ are unknown constants. Imposing the conjugation and boundary conditions, we arrive to a homogeneous system of $m$ linear algebraic equations in the coefficients $C_{j}^{(1)}$, from which one can write a necessary and sufficient condition for the existence of a non-trivial solution of the boundary problem (5)-(77) as follows:

$$
D=\operatorname{det}\left|\sum_{j=1}^{N} u_{j, q}^{(n)} \varphi_{j, s}^{(n)}\left(y_{n}\right)\right|=0,
$$

where the coefficients $u_{j, q}^{(i)}$ for $i=\overline{1, n}$ are given by recurrence; $s, q=\overline{m+1, N}$. Taking into account the dependencies of the functions $\varphi_{j, s}^{(n)}\left(y_{n}\right)$ on the value $\lambda$, we determine the eigenfrequencies of the vibration system as roots of the equation (12).

Given a concrete system, the necessary and sufficient condition (12) provides a general method to investigate the frequency spectrum of possible vibrations and its dependence on the different parameters of the system. This is the main advantage of the numerical method presented here: the application of the normal fundamental functions to an arbitrary but finite number of discrete characteristics is reduced to the determination of zeros of a function, which is 
a determinant; the order of this determinant depends only on the number of the boundary conditions on the integration interval and does not depend on the number of the discrete characteristics in the system; the elements of the determinant are calculated by given recurrence formulas, which is very convenient for numerical solving with computers.

In the next section we apply the proposed method to the study of the eigenvalue problems for some aerial cable-ways.

\section{Eigenvalue Problems for Aerial Cable-Ways}

In this paper we analyze some hybrid controlled systems of aerial cable-ways, which carry some discrete loads. The problem is to study the dynamics of such systems as well as changes of their dynamical characteristics depending on different system parameters and, first of all, depending on the controlled parameter. In this research, the method of the normal fundamental systems described in $\$ 3$ is applied, which gives the best results for this class of eigenvalue problems.

\subsection{The Basic Problem of Transverse Vibrations of the Cable}

First, let us consider only transverse vibrations of the cable. We assume that the cable has a uniform linear density. Then we have the next well-known continuous boundary problem [4]:

$$
\begin{gathered}
\rho \frac{\partial^{2} u}{\partial t^{2}}=T \frac{\partial^{2} u}{\partial x^{2}}, \\
u(0, t)=u(l, t)=0,
\end{gathered}
$$

where $t$ is the time variable; $x$ is the coordinate of some point of the cable; $\rho$ is the mass per unit length; $T$ is the tension in the cable; $l$ is the length of the cable; $u(x, t)$ is the function of deviation from equilibrium position for the point $x \in[0, l]$ at the time moment $t$.

The classical Fourier technique of separation of variables may be used for such simplified problem. We assume that the solution of the equation (13) may be presented as a product of two functions where the first function depends only on the variable $x$ and the second one depends only on the variable $t$, i.e.

$$
u(x, t)=X(x) F(t) .
$$

Then, after separating variables, we will have

$$
\frac{F^{\prime \prime}(t)}{a^{2} F(t)}=\frac{X^{\prime \prime}(x)}{X(x)},
$$


where $a=\sqrt{\frac{T}{\rho}}$. The left-hand side of the relation (16) depends only on $t$ and the right-hand side depends only on $x$. Consequently, each side of this equality is equal to some constant $C$, and the following equations can be written:

$$
\begin{aligned}
& F^{\prime \prime}(t)-a^{2} C F(t)=0, \\
& X^{\prime \prime}(x)-C X(x)=0 .
\end{aligned}
$$

We are interested in finding non-trivial solutions of the equation (18) satisfying boundary conditions

$$
X(0)=X(l)=0,
$$

which obviously follow from the conditions (14).

It is well known 3 that for $C \geq 0$ the equation (18) has only the trivial solution $X(x) \equiv 0$; and that for $C<0$ non-trivial solutions exist. Thus, with the notation $C=-\lambda^{2}$, we can write the equation (18) in the form

$$
X^{\prime \prime}(x)+\lambda^{2} X(x)=0 .
$$

The values $\lambda^{2}$, at which the initial boundary problem (13)-(14) has non-trivial solutions, are called the eigenvalues of this boundary problem. It is easy to show that the eigenvalues may be found from the relationship

$$
\frac{\sin (\lambda l)}{\lambda}=0 .
$$

The general solution of the equation (20), with the accuracy to some constant, is $X(x)=\sin \left(\frac{\pi k x}{l}\right)$ (where $k=1,2, \ldots$ from here forwards), and it takes place at the values $\lambda^{2}=\lambda_{k}^{2}=\frac{\pi^{2} k^{2}}{l^{2}}$, which consequently are the eigenvalues of the boundary problem (13)-114).

Thus, we can see that in our case the eigenvalues $\lambda_{k}^{2}$ are positive and there exists an infinite set of them. Besides, $\lambda_{1}^{2}<\lambda_{2}^{2}<\cdots<\lambda_{k}^{2}<\cdots$ and $\lambda_{\infty}^{2} \rightarrow \infty$. It is found also that $\lambda_{k}^{2} \neq 0$. These important characteristics of the eigenvalues depend on the kind of differential equations, which describe vibrations, and on the boundary conditions. It is necessary to point out that in some cases, for some boundary problems, it is possible to determine qualitative characteristics of the eigenvalues without their numerical calculation.

A particular solution of the equation (13) may be written as follows:

$$
u_{k}(x, t)=X_{k}(x) F_{k}(t)=\sin \left(\frac{\pi k x}{l}\right)\left(\alpha_{k} \cos \left(a \lambda_{k} t\right)+\beta_{k} \sin \left(a \lambda_{k} t\right)\right),
$$

where $\alpha_{k}$ and $\beta_{k}$ are unknown coefficients which have to be determined from the initial conditions of the concrete boundary problem. It is easy to see, from the particular solution (22), that the natural frequencies of vibrations of the considered cable are given by $\omega_{k}=a \lambda_{k}=\frac{\pi k a}{l}, k=1,2, \ldots$ 
Thus, if we know the eigenvalues of the boundary problem (13)-(14), we can always determine its natural frequencies, which are very important physical characteristics of the system under consideration; vice versa, if we know the natural frequencies, we can find the eigenvalues of the problem.

\subsection{The Problem for the Case of Fixed Loads}

Now let us consider the mathematical model of the cable with fixed loads. In this case we have the boundary problem

$$
\begin{gathered}
\rho \frac{\partial^{2} u_{i}}{\partial t^{2}}=T \frac{\partial^{2} u_{i}}{\partial x^{2}}, \\
u_{1}(0, t)=u_{n}(l, t)=0,
\end{gathered}
$$

where $l_{i}$ is the coordinate of the arbitrary point at which the discrete load with the mass $m_{i}$ is fixed, $i=\overline{1, n-1}, l_{0}=0, l_{n}=l ; u_{i}(x, t)$ are the functions of deviation from equilibrium position for the point $x \in\left[l_{i-1}, l_{i}\right]$ at the moment $t, i=\overline{1, n}$. The solution of the problem (23)-(24) must satisfy the following conjugation conditions:

$$
\begin{gathered}
u_{i+1}\left(l_{i}, t\right)=u_{i}\left(l_{i}, t\right), \\
\left.m_{i} \frac{\partial^{2} u_{i}}{\partial t^{2}}\right|_{x=l_{i}}=\left.T\left(\frac{\partial u_{i+1}}{\partial x}-\frac{\partial u_{i}}{\partial x}\right)\right|_{x=l_{i}},
\end{gathered}
$$

where $m_{i}$ are the masses of the discrete loads at arbitrary points $x=l_{i}$ for all $i=\overline{1, n-1}$.

Let us use the numerical method of the normal fundamental system of solutions. An application of this method demands the presentation of the problem as a system of ordinary differential equations of the first order in normal form, satisfying some boundary conditions. In order to reduce (20) to the necessary form, we introduce new functions

$$
T_{i}(x)=X_{i}(x), \quad S_{i}(x)=X_{i}^{\prime}(x),
$$

and write the desired system of differential equations in normal form as

$$
\left\{\begin{array}{l}
T_{i}^{\prime}=S_{i}, \\
S_{i}^{\prime}=-\lambda^{2} T_{i},
\end{array}\right.
$$

with the boundary conditions as follows:

$$
\begin{array}{ll}
\bar{x}=0: & T_{1}=0, \\
\bar{x}=l: & T_{n}=0 .
\end{array}
$$


Using the functions of the normal fundamental system of solutions, we can present the general solution of the equations (20) at the intervals of continuity in the form

$$
X_{i}(x)=A_{i} S_{i}\left(x-l_{i-1}\right)+B_{i} T_{i}\left(x-l_{i-1}\right),
$$

where

$$
S_{i}\left(x-l_{i-1}\right)=\cos \left(\lambda\left(x-l_{i-1}\right)\right), \quad T_{i}\left(x-l_{i-1}\right)=\frac{\sin \left(\lambda\left(x-l_{i-1}\right)\right)}{\lambda},
$$

and $S_{i}^{\prime \prime}=-\lambda^{2} S_{i}, T_{i}^{\prime \prime}=-\lambda^{2} T_{i}$.

In the case of only one discrete load, we obtain the next equation for determination of the eigenvalues:

$$
\frac{\sin (\lambda l)}{\lambda}-\frac{m_{1}}{\rho} \sin \left(\lambda b_{1}\right) \sin \left(\lambda b_{2}\right)=0 .
$$

Comparing the formulas (32) and (21), it is easy to see that the presence of the load at the object decreases the corresponding eigenvalues. If the load is located in the middle of the integration interval, then the equation for determination of the eigenvalues is reduced to

$$
\frac{\sin (\lambda l)}{\lambda}-\frac{m_{1}}{\rho} \sin ^{2}\left(\frac{\lambda l}{2}\right)=0,
$$

where $\frac{m_{1}}{\rho} \gg 1$. From the equations (21), (32) and (33), we have the following properties of the eigenvalues.

Theorem 1. If the discrete mass $m_{1}$ is located in the middle $\frac{l}{2}$ of the integration interval $[0, l]$ of the continuous-discrete boundary problem (23)-(26), then all the even eigenvalues of this problem coincide with the even eigenvalues of the corresponding continuous boundary problem (13)-(14).

Theorem 2. For any location of the discrete mass $m_{1}$ at the integration interval $[0, l]$ of the continuous-discrete boundary problem (23)-(26), the first eigenvalue of this problem is always smaller than the first eigenvalue of the corresponding continuous boundary problem (13)-(14).

Theorem 3. If the continuous-discrete system described by the boundary problem (23) -(26) has only one discrete mass $m_{1}$ which is located in the middle $\frac{l}{2}$ of the integration interval $[0, l]$, then the first eigenvalue of the problem (23)-(26) is the smallest one in comparison with the cases of any other location of the mass $m_{1}$ at the integration interval.

Theorem 3 is in agreement with the physical sense of the process under investigation: if the discrete mass $m_{1}$ is located in the middle of the elastic body, then the amplitude of vibrations on the first frequency at this point is maximal and, consequently, the first eigenvalue and the first frequency of vibrations are minimal. 
In the case of two different discrete masses $m_{1}$ and $m_{2}$, which are arbitrarily located at the cable, we have the next equation to determine the eigenvalues of the problem (23)-(26):

$$
\begin{array}{r}
\frac{1}{\lambda}\left[\sin (\lambda l)-\frac{\lambda m_{1}}{\rho} \sin \left(\lambda b_{1}\right) \sin \left(\lambda\left(b_{1}+b_{2}\right)\right)-\frac{\lambda m_{2}}{\rho} \sin \left(\lambda b_{3}\right) \sin \left(\lambda\left(b_{1}+b_{2}\right)\right)\right. \\
\left.+\frac{\lambda m_{1} m_{2}}{\rho} \sin \left(\lambda b_{1}\right) \sin \left(\lambda b_{2}\right) \sin \left(\lambda b_{3}\right)\right]=0,
\end{array}
$$

where $b_{i}$ is the length of the $i$-th interval of continuity; $i=1,2,3$. At condition $b_{1}=b_{2}=b_{3}$ we can present equation (34) in a more simple form:

$$
\frac{1}{\lambda}\left[\sin (\lambda l)-\frac{2 \lambda\left(m_{1}+m_{2}\right)}{\rho} \sin ^{2}\left(\frac{2 l}{3}\right) \cos \left(\frac{\lambda l}{3}\right)+\frac{\lambda m_{1} m_{2}}{\rho} \sin ^{3}\left(\frac{\lambda l}{3}\right)\right]=0 .
$$

Finally, let us consider the general case of the continuous-discrete boundary problem (23)- (26), when we have $n-1$ discrete masses $m_{i}, i=\overline{1, n-1}$, at arbitrary points of the integration interval $[0, l]$. In this case the determination of the eigenvalues is reduced to finding of zeros of the function

$$
\psi_{n+1}^{(1)}(\lambda)=\psi_{n}^{(1)} S_{n}\left(b_{n}\right)+\psi_{n}^{(2)} T_{n}\left(b_{n}\right),
$$

with $\psi_{i+1}^{(1)}$ and $\psi_{i+1}^{(2)}$ given by the recurrence formulas

$$
\begin{gathered}
\psi_{i+1}^{(1)}=\psi_{i}^{(1)} S_{i}\left(b_{i}\right)+\psi_{i}^{(2)} T_{i}\left(b_{i}\right), \\
\psi_{i+1}^{(2)}=\frac{-\psi_{i}^{(1)}\left[\rho \lambda^{2} T_{i}\left(b_{i}\right)+m_{i} \lambda^{2} S_{i}\left(b_{i}\right)\right]}{\rho} \\
+\frac{\psi_{i}^{(1)}\left[\rho S_{i}\left(b_{i}\right)-m_{i} \lambda^{2} T_{i}\left(b_{i}\right)\right]}{\rho}, \quad i=\overline{1, n-1},
\end{gathered}
$$

where $S_{i}$ and $T_{i}$ are the normal fundamental functions

$$
S_{i}\left(b_{i}\right)=\cos \left(\lambda b_{i}\right), \quad T_{i}\left(b_{i}\right)=\frac{\sin \left(\lambda b_{i}\right)}{\lambda},
$$

$\psi_{1}^{1}$ and $\psi_{1}^{2}$ are known from the left boundary condition (24); $b_{i}$ is the length of the $i$-th interval of continuity, $i=\overline{1, n}$. As a result, we can write the next equation for the determination of the eigenvalues:

$$
\frac{1}{\lambda}\left[\sin (\lambda l)-\lambda f_{n}(\lambda)\right]=0,
$$

where $f_{n}(\lambda)$ includes all functions calculated by the recurrence formulas (37) and (38). From (40) the following property of the eigenvalues can be derived.

Theorem 4. The eigenvalues of the continuous-discrete boundary problem (23)(26) are always less or equal than the eigenvalues of the corresponding continuous boundary problem (13)-(14). 


\subsection{The Problem for the Case When Only the Loads Move}

If only discrete loads with masses $m_{1}, m_{2}, \ldots, m_{n-1}$ move along the cable-way at a steady speed, then the mathematical model for such controlled dynamical system is the next non-stationary boundary problem:

$$
\begin{gathered}
\rho \frac{\partial^{2} u_{i}}{\partial t^{2}}=T \frac{\partial^{2} u_{i}}{\partial x^{2}}, \\
u_{1}(0, t)=u_{n}(l, t)=0,
\end{gathered}
$$

with conjugation conditions

$$
\begin{gathered}
u_{i+1}\left(l_{i}, t\right)=\left.u_{i}\left(l_{i}, t\right)\right|_{x=l_{i}(t)} \\
\left.m_{i}\left(\frac{\partial^{2} u_{i}}{\partial t^{2}}+2 v \frac{\partial^{2} u_{i}}{\partial x \partial t}+v^{2} \frac{\partial^{2} u_{i}}{\partial x^{2}}\right)\right|_{x=l_{i}(t)}=\left.T\left(\frac{\partial u_{i+1}}{\partial x}-\frac{\partial u_{i}}{\partial x}\right)\right|_{x=l_{i}(t)},
\end{gathered}
$$

where $l_{i}(t)=l_{i}+v t, \quad l_{0}(t)=0, \quad t_{0} \leq t \leq t_{1}$.

Let us consider the boundary problem (41)-(44) with only one moving load. Using normal fundamental functions, we obtain the following equation for the determination of non-stationary eigenvalues:

$$
\frac{\sin (\lambda l)}{\lambda}-\frac{m_{1}}{\rho}\left(a^{2}+v^{2}\right) \sin \left(\lambda b_{1}\right) \sin \left(\lambda b_{2}\right)=0,
$$

where $\lambda=\lambda(t) ; \quad b_{1}=l_{1}$ and $b_{2}=l-l_{1}$ at a fixed moment of time. The zeros of the left-hand side of (45) are non-stationary eigenvalues at the time $t_{0} \leq t \leq t_{1}$. It is possible at each fixed moment of time to find non-stationary frequencies $\omega_{n}=\omega_{n}(t)$ at the same interval by the known formula $\omega_{k}=a \lambda_{k}, k=1,2, \ldots$

The role of the speed $v$ as a controlled parameter, which changes the dynamical characteristics of the system, is evident from (45) and (32). The presence of the speed $v$ in (45) implies:

Proposition 1. In the case of one moving load, the eigenvalues and the natural frequencies of the dynamical system (41)-(44) decrease with the controlled parameter $v$, i.e. decrease when the velocity increases.

\subsection{The Mathematical Model for the Case of the Whole System Motion}

Now we assume that the whole system of the aerial cable-way moves at some steady speed. The mathematical model for such hybrid controlled system is a non-stationary boundary problem of second order PDEs. It consists of the equation of motion (46) subject to the boundary conditions (47):

$$
\rho\left(\frac{\partial^{2} u_{i}}{\partial t^{2}}+2 v \frac{\partial^{2} u_{i}}{\partial x \partial t}+v^{2} \frac{\partial^{2} u_{i}}{\partial x^{2}}\right)=T \frac{\partial^{2} u_{i}}{\partial x^{2}}
$$




$$
u_{1}(0, t)=u_{n}(l, t)=0,
$$

where $v$ is the steady speed of the system motion; $l_{i}$ is the initial coordinate of the cable point with the fixed discrete load $m_{i}, i=\overline{1, n-1} ; l_{i}(t)=l_{i}+v t$ is the coordinate at the moment $t$ of the cable point with the fixed discrete load $m_{i}, i=\overline{1, n-1} ; l_{0}(t)=l_{0}=0 ; l_{n}(t)=l_{n}=l ; u_{i}(x, t)$ are the functions of deviation from equilibrium position for the point $x \in\left[l_{i-1}(t), l_{i}(t)\right]$ at the moment $t, i=\overline{1, n}$. Solutions of the problem (46)-(47) must also satisfy the conjugation conditions (48)-(49) at points $x=l_{i}(t)$ :

$$
\begin{gathered}
u_{i+1}\left(l_{i}, t\right)=\left.u_{i}\left(l_{i}, t\right)\right|_{x=l_{i}(t)}, \\
\left.m_{i}\left(\frac{\partial^{2} u_{i}}{\partial t^{2}}+2 v \frac{\partial^{2} u_{i}}{\partial x \partial t}+v^{2} \frac{\partial^{2} u_{i}}{\partial x^{2}}\right)\right|_{x=l_{i}(t)}=\left.T\left(\frac{\partial u_{i+1}}{\partial x}-\frac{\partial u_{i}}{\partial x}\right)\right|_{x=l_{i}(t)},
\end{gathered}
$$

where $m_{1}, m_{2}, \ldots, m_{n-1}$ are the masses of the discrete loads at arbitrary points $x=l_{i}(t), i=\overline{1 ; n-1}$.

Speed $v$, which may be changed within the limits of $0<v \leq v_{1}$, is the controlled parameter for this real dynamical system. The role of the speed $v$ may be shown for the problem (46)-(49) even in the case of absence of discrete loads.

If in the equation (46) we do not neglect the Coriolis acceleration $\frac{\partial^{2} u}{\partial x \partial t}$, then the natural frequency is determined as follows:

$$
\omega_{k}=\frac{\pi k}{l} \frac{a^{2}-v^{2}}{a}, \quad a>v .
$$

In the case when we do not take into account the Coriolis acceleration in this boundary problem, the natural frequencies are given by

$$
\omega_{k}=\frac{\pi k}{l} \sqrt{\frac{a^{2}-v^{2}}{a}}, \quad a>v .
$$

From (501), (51) one can see that the speed $v$ may significantly decrease the natural frequencies. Here, the obtained frequencies, in contrast to the problems considered below, do not depend on time but depend on the speed only.

Besides, if we consider the case when the interval of integration changes with a constant velocity, then the natural frequency for the given time interval $t_{0} \leq t \leq t_{1}$ may be determined from the formula

$$
\omega_{k}(t)=\frac{\pi k}{l(t)} \frac{a^{2}-v^{2}}{a}, \quad a>v
$$

when the Coriolis acceleration is taken into account, or by

$$
\omega_{k}(t)=\frac{\pi k}{l(t)} \sqrt{\frac{a^{2}-v^{2}}{a}}, \quad a>v,
$$

if the Coriolis acceleration is neglected. From the formulas (52) and (53) we get the following result. 
Theorem 5. When the interval of integration changes with a constant velocity, the speed $v$ of the cable motion decreases the frequencies of vibrations for problem (46) - (49). Also, the decrease of the integration interval, under the condition of invariance of the initial tension, always increase the frequencies.

\section{Conclusions}

In this paper we consider several eigenvalue problems that model the dynamics of some hybrid systems of aerial cable-ways with different assumptions. We begin with an overview of the methods found in the literature for similar eigenvalue problems. The method of the normal fundamental systems turns out to be the most effective one for the considered problems. This method is applied, and the dependence of the dynamical characteristics of the systems on the controlled parameter is established. However, many open problems still exist in this field. First of all, the most general eigenvalue problem (46)-(47) is not studied for the case of presence of the discrete loads. Besides, for many applications it is important to consider systems where the masses of the discrete loads are decreasing with time [4. All these problems are supposed to be solved by the same method in future investigations.

\section{Acknowledgements}

The first author is grateful to the partial financial support provided by the Control Theory Group (cotg) of the Center for Research in Optimization and Control (CEOC) of the University of Aveiro, for participation in The International Conference of Hybrid Systems and Applications, held at the University of Louisiana, Lafayette, LA, USA, May 22-26, 2006. The hospitality and the good working conditions at the University of Aveiro are also gratefully acknowledged. Also the authors are grateful to Enrique H. Manfredini for the suggestions regarding improvement of the text.

\section{References}

[1] L. D. Akulenko, S. V. Nesterov, High-precision methods in eigenvalue problems and their applications, Differential and Integral Equations and Their Applications, 6, Chapman \& Hall/CRC, Boca Raton, FL, 2005, 239 pp.

[2] S. H. Gould, Variational Methods for Eigenvalue Problems, (An introduction to the methods of Rayleigh, Ritz, Weinstein, and Aronszajn. Reprint of the 1957 original), Dover Publications, Inc., New York, 1995, 179 pp.

[3] K. Ya. Kuhta, V. P. Kravchenko, Normalnye fundamentalnye sistemy v zadachakh teorii kolebanij, (Russian) [Normal Fundamental Systems in Vibration Theory Problems], Naukova dumka, Kiev, 1973, 205 pp. 
[4] K. Ya. Kukhta, V. P. Kravchenko, V. A. Krasnoshapka, Kachestvennaya teoriya upravlyaemykh dinamicheskikh sistem $s$ nepreryvno-diskretnymi parametrami, (Russian) [The qualitative theory of controllable dynamical systems with continuous-discrete parameters], Naukova Dumka, Kiev, 1986, 223 pp.

[5] D. Motreanu, V. Rădulescu, Variational and non-variational methods in nonlinear analysis and boundary value problems, Nonconvex Optimization and its Applications, 67, Kluwer Academic Publishers, Dordrecht, 2003, 375 pp.

[6] E. V. Mul, On Conditions of Excitation of Self-Oscillations in a Nonconservative Dynamic System with Distributed Parameters, Cybernetics and Computing Technology, Complex Control Systems, Allerton Press Inc., New York, 1998, vol. 111, 70-72.

[7] E. Mul, V. Kravchenko, Investigations of Vibrations in the Complex Dynamical Systems of Transmission Pipelines, Lecture Notes in Computational Science and Engineering, Springer-Verlag Berlin Heidelberg, 2003, vol.32 "Interface and Transport Dynamics. Computational Modelling", $295-300$.

[8] O. V. Mul, D. F. M. Torres, Analysis of Vibrations in Large Flexible Hybrid Systems, Nonlinear Analysis, Elsevier, 2005, vol. 63, no. 3, 350-363.

[9] O. V. Mul, D. F. M. Torres, Some Applications of the Method of Normal Fundamental Functions to Oscillation Problems, in Proceedings of MTNS 2006 (the 17th International Symposium on Mathematical Theory of Networks and Systems), July 24-28, 2006, Kyoto, Japan [to appear].

[10] J. M. Sloss, I. S. Sadek, J. C. Jr. Bruch, S. Adali, Optimal control of structural dynamic systems in one space dimension using a maximum principle, J. Vib. Control, 2005, vol. 11, no. 2, 245-261. 